\title{
Die Schwierigkeiten mit der Durchimpfung
}

\author{
Dr. med. Daniel Koch ist Leiter der Sektion Impfungen im Bundesamt für Gesundheit in Bern, Schweiz
}

Eigentlich wäre es sehr einfach: Die verantwortlichen und verantwortungsbewussten Behörden empfehlen zum Wohle der Bevölkerung und nach gründlichem Studium aller massgeblichen Fakten eine Impfung und der gut informierte Bürger identifiziert sich mit den Argumenten und lässt sich oder seine Kinder daher impfen. In einer solch idealen Welt könnte man ohne Probleme auf die Überwachung der Durchimpfung verzichten, die gesetzten Ziele der Kontrolle oder gar Elimination von Krankheiten würden zweifelsfrei erreicht. Um wie vieles komplexer und schwieriger die Realität jedoch ist, wurde erst kürzlich in einem Meeting in Paris wieder deutlich. Wärend zwei Tagen diskutierten Vertreter europäischer Gesundheitsbehörden unter der Regie der Weltgesundheitsorganisation (WHO) die Probleme der Masernelimination. Dabei ging es einmal nicht um die Schwierigkeiten auf dem afrikanischen oder asiatischen Kontinent, wo trotz aller Bemühungen die finanziellen Mittel nicht selten kaum zum Kauf von genügend Impfstoff ausreichen, sondern es ging um die Schwierigkeit der reichen westeuropäischen Länder, die Masern in Europa zu eliminieren. Verschiedene nördliche und östliche europäische Länder, der ganze amerikanische Kontinent, aber auch Spanien sind weitgehend masernfrei und werden in erster Linie durch eingeführte Masernviren gefährdet. Die Schweiz. gehört leider zum unrühmlichen, kleinen, westeuropäischen Grüpplein der masernexportierenden Länder, zusammen mit den Nachbarn Deutschland, Österreich, Frankreich und Italien. Ohne ein klares Bild der Durchimpfungsraten können die Probleme, welche zu dieser untragbaren Situation führen, nicht angegangen werden. Beobachtungen über Zeitverläufe, wie sie in Genf durchgeführt werden, sind von unschätzbarem Wert und bilden die Grundlage für zielgerechte Strategien, die Durchimpfungsraten anzuheben, sofern der politische Wille und die nötigen Mittel dies erlauben. Das es aber gerade im deutschsprachigen Raum besonders schwierig ist, akzeptable Durchimpfungraten zu erreichen, zeigt sich leider auch am Beispiel der tiefen Influenza-

Impfraten beim Krankenhauspersonal in Deutschland. Offensichtlich ist es sehr schwierig, in unseren stark dezentralisierten, enorm komplexen Gesundheitssystemen klare, verbindliche Ziele der öffentlichen Gesundheit zu erreichen. Einzelne Interessensgruppen können aus unterschiedlichsten Gründen solche Ziele erfolgreich in Frage stellen und das Erreichen verhindern. Sicher ist es ohnehin viel einfacher, Zweifel zu streuen und Behauptungen aufzustellen, als wissenschaftliche Beweise zu erbringen. Wenn dann aber ausserdem Organisationen wie der Konsumentenschutz oder gar AEGIS die Ziele der öffentlichen Gesundheit torpedieren, dann werden wissenschaftliche Fragen zum Politikum und die sachliche Information bleibt auf der Strecke. Trotz allem müssen das Wohl und die Gesundheit der Schwächsten bei den Behörden weiterhin höchste Priorität haben. Deshalb darf man Ziele wie eine hohe InfluenzaDurchimpfungsrate beim Pflegepersonal, welche den Schutz. von Patienten und Heimbewohnern anstrebt, oder die Masernelimination, welche den vollständigen Schutz der Kinder erreicht, nicht einfach fallen lassen. Sachliche Information, professionelles Engagement und Bereitstellen von Infrastrukturen sind die Mittel, welche schlussendlich zum Wohle der öffentlichen Gesundheit bei diesen Problemen zum Ziel führen werden.

Daniel Koch

Korrespondenzadresse
Dr. Daniel Koch
Bundesamt für Gesundheit
Leiter Sektion Impfungen
CH-3003 Bern
e-mail: daniel.koch@bag.admin.ch 